\title{
Fast MIMO-ISAR Back Projection Algorithm Based on FFT
}

\author{
Wang Xiuyou ${ }^{1, a}$,Wang Hao ${ }^{1 . b}$,Xu Dongqing ${ }^{1 . c}$,Liu Huaming ${ }^{1, d}$,Fan \\ Jianzhong ${ }^{1 . e}$ \\ ${ }^{1}$ School of Computer and Information Engineering, Fuyang Teachers College, Fuyang Anhui \\ 236037, China \\ awangxiuyou@163.com, bahseal@163.com, '9886114346@qq.com, dliuhuaming888@126.com \\ , ${ }^{\prime} f j z$ 73@126.com
}

Keywords: MIMO-ISAR, back projection, fast algorithm

\begin{abstract}
In order to reduce the computational cost in the current MIMO-ISAR back projection imaging algorithm, a fast algorithm based on FFT is proposed for MIMO-ISAR back projection. First, we establish the reflected wave signal model for MIMO-ISAR imaging. The approximate representation of linear change of phase compensation factor is provided. Second, the constraints of the conversion from non-linear phase compensation factor to linear compensation factor is given. Finally, an efficient algorithm for MIMO-ISAR back projection based on FFT is achieved. Simulation results show that compared with traditional methods, the proposed algorithm reduces the computational cost greatly.
\end{abstract}

\section{Introduction}

Inverse Synthetic Aperture Radar (ISAR) can achieve the accurate information of the target pose and structure, which is important for classification and recognition [1,2]. However, the ISAR imaging is based on the relative movement between radar and target, the unexpected movement of the target may cause many issues in imaging process. For example, for the minor movement of the target, ISAR vertical resolution is low, for the large movement, the complicated compensation is difficult [3].

In order to address this problem, MIMO radar and ISAR technology is combined, which is known as MIMO-ISAR [4-7]. Reference [8] proposed to use a re-ranking spatial time method based on two-dimensional frequency estimation in MIMO ISAR. Reference [9] studied the motion parameter estimation in rotation target with constant acceleration. In their work, a joint estimation of initial angle and rotation acceleration was proposed and analyzed.

Back Projection Algorithm (BPA) is an accurate time-domain algorithm. It better adapted to uneven data from MIMO-ISAR. The imaging process is simple and the re-ranking and interpolation are not needed. However, BP algorithm requires a large computational resources, which is the major drawback. Reference [10] proposed an improved BA algorithm based on focusing process of the same distance unit. It has improved the overall efficiency but computational cost with the same distance unit is still high. Reference [11] proposed fast factor decomposition which can reduce the computational cost. However, it can not be applied to MIMO-ISAR directly. In this paper, we consider the property of the MIMO-ISAR and make use of the far field condition for approximation. We propose a fast BP algorithm based on FFT, and give the constraint conditions. The computational cost is analyzed and the effectiveness is verified in experiments.

\section{Return Signal Model}

In this paper we consider MIMO linear array, the target velocity $V$ is parallel with the array direction, as shown in Fig.1. The number of transmitting array element is $M$, the number of receiving array element is $N$, X-coordinates are $u_{m}(m=1,2,3, \cdots, M)$ and $v_{n}(n=1,2,3, \cdots, N)$. Suppose there exists a target in the far field, $Q$ is any point on the target, take polar coordinates representation form with $O$ as the origin: $\left(r_{q}, \theta_{q}\right)$. The scattering parameter is $\xi_{q}, O^{\prime}$ is the center point of the imaging 
scene with coordinates: $\left(r_{0}, \theta_{0}\right)$, and the sector in Fig.1 is the imaging segmentation area.

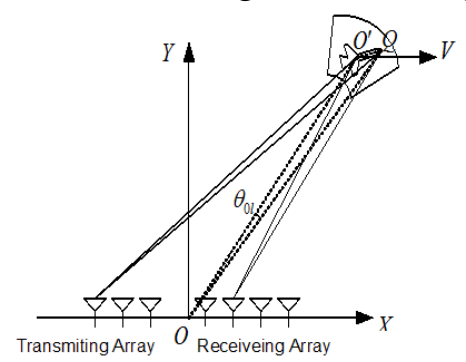

Fig.1. A depiction of MIMO-ISAR imaging

\section{BP Imaging Algorithm Based on FFT}

For high resolution target imaging and low computational cost. We propose to use FFT to develop the fast BP algorithm for MIMO-ISAR. According to Fig.1, separate the target area as $K \times L$ in the polar coordinates with $O$ as the origin. $K$ is the number of the points in distance segment. $L$ is the number of points in angle segment. A pixel is represented as: $\left(r_{k}, \theta_{l}\right),(k=1,2, \cdots, K ; l=1,2, \cdots, L)$. Round trip delay time between Pixel $\left(r_{k}, \theta_{l}\right)$ and any array combination is:

$$
\tau_{m n}^{(k, l)}\left(t_{p}\right)=\frac{\left(\sqrt{\left(r_{k} \sin \left(\theta_{l}\right)-\left(u_{m}-V t_{p}\right)\right)^{2}+\left(r_{k} \cos \left(\theta_{l}\right)\right)^{2}}+\sqrt{\left(r_{k} \sin \left(\theta_{l}\right)-\left(v_{n}-V t_{p}\right)\right)^{2}+\left(r_{k} \cos \left(\theta_{l}\right)\right)^{2}}\right)}{c} .
$$

where $V$ is the target velocity, $t_{p}$ is slow time. Suppose the target can be estimated accurately, perform the distance compensation[10], and the result of BP algorithm imaging is:

$$
I(k, l)=\sum_{m=1}^{M} \sum_{n=1}^{N} \sum_{p=1}^{P} y_{m n}\left(\hat{t}, t_{p}\right) \cdot \exp \left(j 2 \pi f_{c} \tau_{m q n}^{(k, l)}\left(t_{p}\right)\right) \cdot
$$

$y_{m n}\left(\hat{t}, t_{p}\right)$ is the imaging data with orthogonal distance. Perform Taylor series expansion on Eq.(1), taking out the second order component we have:

$$
\tau_{m n}^{(k, l)}\left(t_{p}\right) \approx \frac{R_{m 0}^{(k, l)}-\left(\frac{u_{m}}{R_{m 0}^{(k, l)}}-\frac{r_{k}}{R_{m 0}^{(k, l)}} \sin \left(\theta_{l}\right)\right) V t_{p}+R_{n 0}^{(k, l)}-\left(\frac{v_{n}}{R_{n 0}^{(k, l)}}-\frac{r_{k}}{R_{n 0}^{(k, l)}} \sin \left(\theta_{l}\right)\right) V t_{p}}{C} .
$$

where $R_{m 0}^{(k, l)}=\sqrt{\left(r_{k} \sin \left(\theta_{l}\right)-u_{m}\right)^{2}+\left(r_{k} \cos \left(\theta_{l}\right)\right)^{2}}, \quad R_{n 0}^{(k, l)}=\sqrt{\left(r_{k} \sin \left(\theta_{l}\right)-v_{n}\right)^{2}+\left(r_{k} \cos \left(\theta_{l}\right)\right)^{2}}$.

When the target is located at the far field, $R_{m 0}^{(k, l)} \gg u_{m}, R_{n 0}^{(k, l)} \gg v_{n}$, we have $R_{m 0}^{(k, l)} \approx R_{m 0}, R_{n 0}^{(k, l)} \approx R_{n 0}$, and $r_{k} / R_{m 0}^{(k, l)} \approx r_{k} / R_{n 0}^{(k, l)} \approx 1$. Equation (3) can be approximately represented as:

$$
\tau_{m n}^{(k, l)}\left(t_{p}\right) \approx \frac{R_{m 0}^{(k, l)}+\left(\frac{u_{m}}{R_{m 0}}-\sin \left(\theta_{l}\right)\right) V t_{p}+R_{n 0}^{(k, l)}+\left(\frac{v_{n}}{R_{n 0}}-\sin \left(\theta_{l}\right)\right) V t_{p}}{c} .
$$

where $R_{m 0}=\sqrt{\left(r_{0} \sin \left(\theta_{0}\right)-u_{m}\right)^{2}+\left(r_{0} \cos \left(\theta_{0}\right)\right)^{2}}, \quad R_{n 0}=\sqrt{\left(r_{0} \sin \left(\theta_{0}\right)-u_{m}\right)^{2}+\left(r_{0} \cos \left(\theta_{0}\right)\right)^{2}}$.

Submit Eq.(4) to Eq.(2), we have:

$$
\begin{aligned}
I(k, l)= & \sum_{m=1}^{M} \sum_{n=1}^{N} \exp \left(j \frac{4 \pi}{\lambda}\left(R_{m 0}^{(k, l)}+R_{n 0}^{(k, l)}\right)\right) \sum_{p=1}^{P} y_{m n}\left(\hat{t}, t_{p}\right) . \\
& \cdot \exp \left(j \frac{4 \pi}{\lambda}\left(\frac{u_{m}}{R_{m 0}}+\frac{v_{n}}{R_{n 0}}-2 \sin \left(\theta_{l}\right)\right) V t_{p}\right)
\end{aligned}
$$

where $\lambda$ is the wave length. At the far field $\sin \left(\theta_{l}\right) \approx \theta_{l}$, let $f_{m n d}=\left(\frac{u_{m}}{R_{m 0}}+\frac{v_{n}}{R_{n 0}}-2 \theta_{l}\right) V$, Eq.(5) can be 
simplified as:

$$
I(k, l)=\sum_{m=1}^{M} \sum_{n=1}^{N} \exp \left(j \frac{4 \pi}{\lambda}\left(R_{m 0}^{(k, l)}+R_{n 0}^{(k, l)}\right)\right) \sum_{p=1}^{P} y_{m n}\left(\hat{t}, t_{p}\right) \cdot \exp \left(j \frac{4 \pi}{\lambda} f_{m n d} t_{p}\right) .
$$

According to Eq.(6), the first exponential component is only related to $m, n$. For fixed transmitting and receiving array, the second component is related to $t_{p}$ and $\theta$. The second sum is in fact the Fourier transform. Therefore, we can use FFT to perform fast computing. Based on Eq.(6), the fast BP imaging algorithms are represented as: Step 1: Use the distance correction method in [10] to compensate the imaging data; Step 2: Separate the imaging areas, construct the Fourier transform factor for each set of data; Step 3: Use FFT to calculate the third component in Eq.(6) and get the low resolution target; Step 4: Fine segmenting the imaging area, use the result from Step 2 to process each pixel for high resolution image.

\section{Algorithm Analysis}

Based on the Sec.3, FFT can be used for the calculation and the focusing process can be improved. However, there are still two remaining questions: one is the constraint conditions in the approximation, the other is the computation cost. Next we will address the above two questions.

The purpose of the approximation is the replace the accurate phase compensation factor with the approximate factor. The phase factor should satisfy the coherent property. The error of the phase estimation should be smaller than $\pi / 4[12]$. According to Eq.(1) and Eq.(6), the difference in the approximate phase compensation factor in $\mathrm{BP}$ algorithm is:

$$
\begin{aligned}
& \Delta \phi=-\frac{4 \pi}{\lambda}\left(\sqrt{\left(r_{k} \sin \left(\theta_{l}\right)-\left(u_{m}+V t_{p}\right)\right)^{2}+\left(r_{k} \cos \left(\theta_{l}\right)\right)^{2}}\right. \\
& +\sqrt{\left(r_{k} \sin \left(\theta_{l}\right)-\left(v_{n}+V t_{p}\right)\right)^{2}+\left(r_{k} \cos \left(\theta_{l}\right)\right)^{2}} \\
& \left.-R_{m 0}^{(k, l)}+\left(\frac{u_{m}}{R_{m 0}}-\sin \left(\theta_{l}\right)\right) V t_{p}-R_{n 0}^{(k, l)}+\left(\frac{v_{n}}{R_{n 0}}-\sin \left(\theta_{l}\right)\right) V t_{p}\right)
\end{aligned}
$$

Simplify Eq.(7) with Taylor series expansion:

$$
\begin{aligned}
\Delta \phi & =-\frac{4 \pi}{\lambda}\left(\left(\frac{u_{m}}{R_{m 0}^{(k, l)}}-\frac{u_{m}}{R_{m 0}}\right) V t_{p}+\left(\frac{v_{n}}{R_{n 0}^{(k, l)}}-\frac{v_{n}}{R_{n 0}}\right) V t_{p}\right. \\
& -\left(\frac{r_{k}}{R_{m 0}^{k, l)}} \sin \left(\theta_{l}\right)-\theta_{l}\right) V t_{p}-\left(\frac{r_{k}}{R_{n 0}^{(k, l)}} \sin \left(\theta_{l}\right)-\theta_{l}\right) V t_{p} \\
& \left.+\sum_{i=2}^{\infty} a_{i} \cdot \frac{d^{(i)} R_{m 0}^{(k, l)}}{d t_{p}^{i}} \cdot t_{p}^{i}+\sum_{i=2}^{\infty} b_{i} \cdot \frac{d^{(i)} R_{n 0}^{(k, l)}}{d t_{p}^{i}} \cdot t_{p}^{i}\right)
\end{aligned}
$$

where the $i$-th order derivative is:

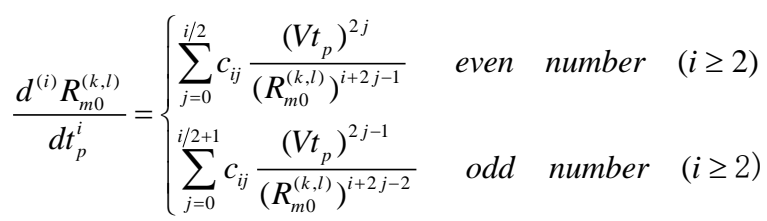

where $c_{i j}$ is the coefficient, then we have:

$$
\frac{4 \pi}{\lambda} \cdot\left(\sum_{i=2}^{\infty} a_{i} \cdot \frac{d^{(i)} R_{m 0}^{(k, l)}}{d t_{p}^{i}} \cdot t_{p}^{i}+\sum_{i=2}^{\infty} b_{i} \cdot \frac{d^{(i)} R_{n 0}^{(k, l)}}{d t_{p}^{i}} \cdot t_{p}^{i}\right) \ll \frac{\pi}{4} .
$$

We only need to consider the following constraint:

$$
\begin{aligned}
& \frac{4 \pi}{\lambda}\left[\left(\frac{u_{m}}{R_{m 0}^{(k, l)}}-\frac{u_{m}}{R_{m 0}}\right)+\left(\frac{v_{n}}{R_{n 0}^{(k, l)}}-\frac{v_{n}}{R_{n 0}}\right)-\left(\frac{r_{k}}{R_{m 0}^{(k, l)}} \sin \left(\theta_{l}\right)-\theta_{l}\right) .\right. \\
& \left.-\left(\frac{r_{k}}{R_{n 0}^{(k, l)}} \sin \left(\theta_{l}\right)-\theta_{l}\right)\right] V T_{p}<\frac{\pi}{4}
\end{aligned} .
$$


where $T_{p}$ is the accumulated time, let $d_{p}=V T_{p}$, we have:

$$
d_{p}<\frac{\lambda}{16 \cdot\left(u_{m} \frac{R_{m 0}-R_{m 0}^{(k, l)}}{R_{m 0}^{(k, l)} R_{m 0}}+v_{n} \frac{R_{n 0}-R_{n 0}^{(k, l)}}{R_{n 0}^{(k, l)} R_{n 0}}-\frac{r_{k}\left(R_{m 0}^{(k, l)}+R_{n 0}^{(k, l)}\right)}{R_{m 0}^{(k, l)} R_{n 0}^{(k, l)}} \sin \left(\theta_{l}\right)+2 \theta_{l}\right)}
$$

Eq. (12) is the constraint condition of our method.

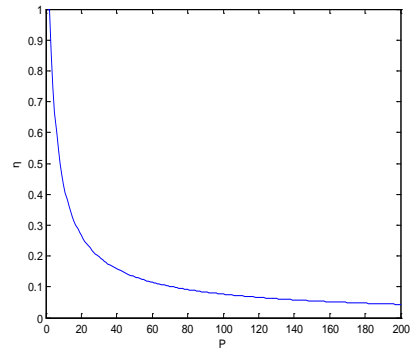

Fig. 2. The change of computational ratio

Our distance compensation method is the same as reference [10], and the computational cost is also the same. For simplicity, we only consider the coherent computation cost. The number of complex multiplications in reference [10] is $M \times N \times K \times L \times P$, our method is: $M \times N \times K \times L \times\left(1+\log _{2} P\right)$. The ratio of the two methods is:

$$
\eta=\frac{1+\log _{2} P}{P} \text {. }
$$

Consider Eq.(13), when $P>1, \eta<1$, along with the increase of $P, \eta$ decrease very fast converging to zero. The relation curve is shown in Fig.2. From Fig.2 we can see that our algorithm can improve the computational efficiency significantly. When $P$ rises and $\eta$ decreases, the computational efficiency is better improved.

\section{Simulation and Analysis}

The parameter settings are: 3-transmitting and 8-receiving MIMO array is used. The coordinates of the transmitting array are: $(-300,0)(-180,0)$ and $(-60,0)$. The coordinates of the transmitting array are: $(60,0)(75,0)(90,0)(105,0)(120,0)(135,0)(150,0)$ and $(165,0)$. Radar parameters are shown in Table 1.

Table1. Radar parameter

\begin{tabular}{lr}
\hline Carrier & $10 \mathrm{GHz}$ \\
Signal Form & Phase Encoding \\
Bandwidth & $150 \mathrm{MHz}$ \\
Sampling Rate & $1 \mathrm{GHz}$ \\
Impulse Width & $80 \mathrm{~ns}$ \\
Sub-Impulse Width & $2 \mathrm{~ns}$ \\
Impulse Repeating Frequency & $400 \mathrm{~Hz}$ \\
Impulse Accumulated Time & $0.1 \mathrm{~s}$
\end{tabular}

Simulation 1: based on the above array and radar parameter settings, simulate the multiple scattering points imaging. The target contains 9 points, which is shown in Fig.3.The distance from the target center to the array center is $R_{0}=10000 \mathrm{~m}$. The target moving in a straight line with uniform velocity: $V=150 \mathrm{~m} / \mathrm{s}$. Suppose the error of the estimation of the velocity is: $0.1 \mathrm{~m} / \mathrm{s}$, imaging result is shown in Fig.4.

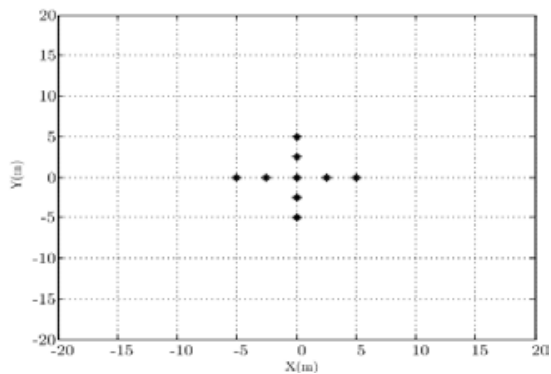

Fig.3. Locations of target scattering points 


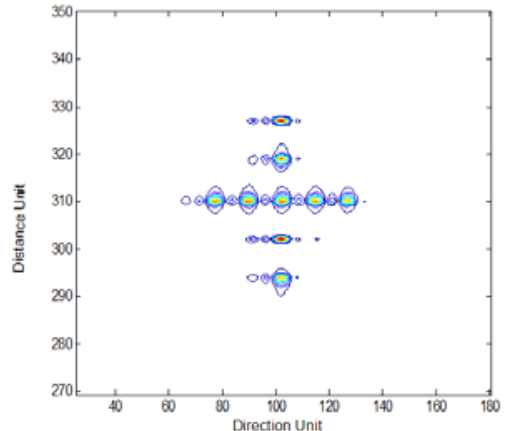

(a) Imaging result from reference [10]

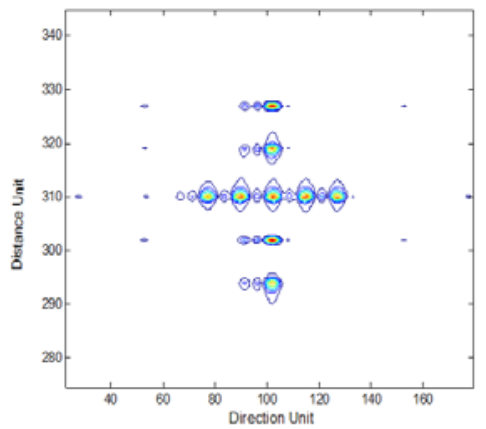

(b)Imaging result from our algorithm

Fig.4. Comparison of imaging results

In the simulation, the computational time records are 43.5379s and 6.9242s for reference [10] and our algorithm respectively. Fig. 4 is the comparison of two algorithms. The image entropies are 9.0076 and 9.1239. From the results we can see that our algorithm effectively reduced the cost although the fake point is introduced in the imaging result. The side lobe is increased, and the entropy is also increased. Comparing Fig.4 (a) and (b), we can see that the fake point is not impacting the image quality significantly. Therefore, our algorithm can improve the computational efficiency while maintaining high image quality.

From the analysis in Sec.3, we can see that the computational efficiency is higher when $P$ is higher. Based on the constraint in Eq.(12) the rising of $P$ will cause the rising of $d_{p}$ and the error in phase compensation also rises. In order to further explore the relation between the computational efficiency and the imaging quality, we compare the point spreading function of the single scattering point and the side lobe in simulation 2.

Simulation 2: based on the above array and radar parameter, carry out simulation on the signal scattering point. The point coordinate is: $(10000,0)$, with velocity $V=150 \mathrm{~m} / \mathrm{s}$. Suppose the estimation error of the velocity is: $0.1 \mathrm{~m} / \mathrm{s}$, simulation results are shown in Fig.5 and Fig.6.
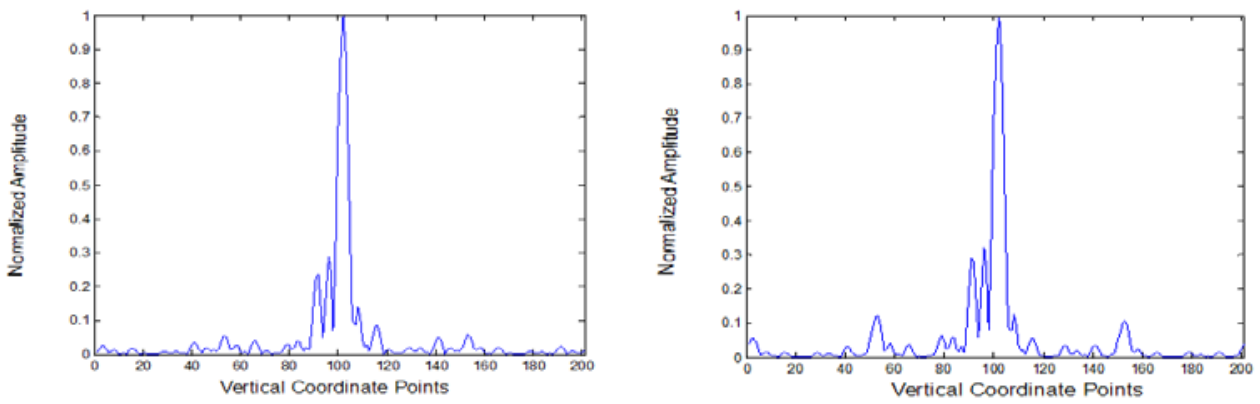

(a) Point spreading function from reference (b) Point spreading function from our method

Figure 5. Comparison of point spreading function

Fig. 5 is the point spreading function of two algorithms. Comparing Fig.5 (a) and (b), the main lobes are consistent. The side lobe of our algorithm is raised. According to the simulation we can get the peak side-lobe level ratio (PSLR) are -11.06dB and -9.9dB.

Fig.6 is the relation curve about the PSLR and the computational efficiency. As $\eta$ decreases, the computational efficiency is improved and the PSLR increases. And when the PSLR requirement is met, the proposed algorithm can improve the efficiency of BP algorithm.

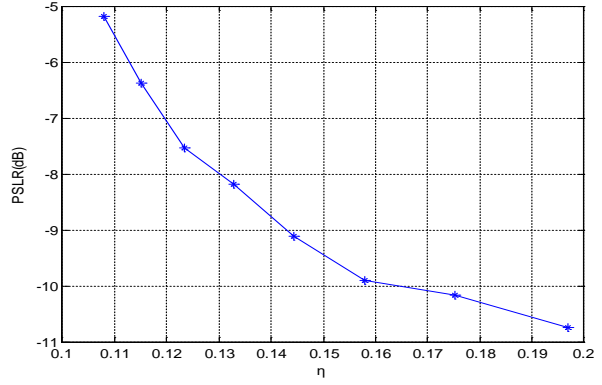

Fig.6. Relation between the PSLR and computational cost 


\section{Conclusion}

In this paper we study the computational cost in MIMO-ISAR back projection imaging. Under the far filed condition we take reasonable approximation of phase factor and propose a fast BP algorithm of MIMO-ISAR based on FFT. We also give the constraint condition of converting the non-linear phase compensation factor to the linear phase compensation factor. The experimental results show that the proposed algorithm decrease the computation time and maintained a high imaging quality. In future work we may further improve the phase mismatch and side lobe change.

\section{Acknowledgement}

This paper is supported by the key project of Natural Science Research of universities in Anhui Province(KJ2016A561).

\section{Reference}

[1] Huang Ya-jing, Wang Xue-zhi, Li Xiang, et al. Inverse synthetic aperture radar imaging using frame theory[J]. IEEE

Transactions on Signal Processing, 2012, 60(5): 5191-5200.

[2] Bai Xue-ru, Zhou Feng, Xing Meng-dao, et al. Highresolution radar imaging of air targets from sparse azimuth data[J]. IEEE Transactions on Aerospace and Electronic Systems, 2012, 48(2): 1643-1655.

[3] Ye Chun-mao, Xu Jia, Peng Ying-ning, et al.. Cross-range scaling for isar via optical flow analysis[J]. IEEE Aerospace and Electronic Systems Magazine, 2012, 27(2): 14-22.

[4] Zhu Yu-tao, Yu Wen-xian, Su Yi. An ISAR Imaging Method Based on MIMO Technique[J].Acta Electronic Asinica, 2009, 37(4):1885-1894.

[5] Debora Pastina, Fabrizio Santi, and Marta Bucciarelli. Multistatic and MIMO Distributed ISAR for Enhanced Cross-Range Resolution of Rotating Targets[J]. IEEE Transactions on Geoscience and Remote Sensing, 2010, 48(3):3300-3316.

[6] Debora Pastina, Fabrizio Santi, and Marta Bucciarelli. MIMO Distributed Imaging of Rotating Targets for Improved 2-D Resolution [J]. IEEE Geoscience and Remote Sensing Ltters, 2015, 12(1):190-194.

[7] Zhu Yu-tao, Su Yi, and Yu Wenxian. An ISAR Imaging Method Based on MIMO Technique[J]. IEEE Transactions on Geoscience and Remote Sensing, 2010, 48(3):3290-3299.

[8] Yang Jian-chao, Su Wei-min, Gu Hong. A Method for Rearrangement of 2D MIMO-ISAR Space-time Echo Based on 2D Frequency Estimation[J].Journal of Electronics \& Information Technology, 2014,36(4):2180-2186.

[9] Chen Gang, GU Hong, SU Wei-min. Motion Parameter Estimation and Performance Analysis for Constant Accelerating Rotating Target in MIMO-ISAR Imaging[J].Journal of Electronics \& Information Technology, 2014,36(4):1919-1925.

[10] Dong Hui-xu, Zhang Yong-shun, Feng Cun-qian, et al. Two-Dimensional Imaging Using MIMO Radar and ISAR Technique Based on Linear Array[J].Journal of Electronics \& Information Technology, 2015,37(2):309-314.

[11] Ulander L M H, Hellsten H, Stenstrom G. Synthetic-aperture radar processing using fast factorized back-projection[J]. IEEE Transactions on Aerospace and Electronic Systems, 2003,39(3):760-776.

[12] Bao Zheng, Xing Meng-dao,Wang Tong. Radar imaging technology[M]. Bei-jing: Publishing House of Electronics Industry, 2005. 